\title{
Properties of Graphene Oxide/Epoxy Resin Composites
}

\author{
Jijun Tang, Haijun Zhou, Yunxia Liang, Xinlan Shi, Xin Yang, and Jiaoxia Zhang
}

School of Materials Science and Engineering, Jiangsu University of Science and Technology, Zhenjiang 212003, China

Correspondence should be addressed to Haijun Zhou; zhjjx@163.com and Jiaoxia Zhang; myzjx0359@163.com

Received 19 July 2014; Accepted 14 September 2014; Published 6 November 2014

Academic Editor: Xiao-Miao Feng

Copyright (C) 2014 Jijun Tang et al. This is an open access article distributed under the Creative Commons Attribution License, which permits unrestricted use, distribution, and reproduction in any medium, provided the original work is properly cited.

\begin{abstract}
The graphene oxide (GO) was obtained by pressurized oxidation method using natural graphite as raw materials. Then the GO/epoxy resin composites were prepared by casting. The mechanical and damping properties of composites were studied. As a result, the impact intensity of GO/epoxy resin composites was prominently improved with the content of the graphene oxide increasing. The glass transition temperature decreased and the damping capacity is improved.
\end{abstract}

\section{Introduction}

In the narrow sense, graphene refers to the single sheet of graphite just $0.335 \mathrm{~nm}$ thick, with the carbon atoms arranged in a honeycomb lattice. It can be seen as infinite aromatic molecules, $\mathrm{sp}^{2}$ hybridized carbons' bond length is only about $1.42 \AA$. Broadly speaking, graphite with less than 10 layers can be called graphene. Graphene is the basic structural unit of carbon materials like carbon nanotubes, graphite, fullerene, and so forth $[1,2]$. It has become a research hotspot of materials science and coacervation physics in physics research and practical application since it was discovered in 2004 due to its excellent physical and chemical properties [3]. But the interaction between pure graphene and other media is weak and there are strong van der Waals forces between graphene sheets, which make graphene prone to aggregate; thus, it is difficult to give full scope to its superior performance. Graphene oxide has similar structure with graphene, hydroxyl and epoxy are located on the basal plane of graphene oxide, carbonyl, and carboxyl mainly in the edge of the graphene oxide, and these characteristics give graphene oxide satisfactory composite capability and improve the dispersion of graphene oxide in the matrix resin $[4,5]$. Epoxy resins are used in a variety of applications because of their properties, such as thermal stability, mechanical response, low density, and electrical resistance, but low tenacity and destruction resistant ability restrict its use. Liang et al. [6] dispersed graphene in the epoxy resin to prepare the composite material. The research shows that the electromagnetic obstacle resistance effect of the prepared material significantly improved when the quality ratio of graphene and epoxy resin was $3: 20$. Bortz et al. [7] prepared the composite materials with functional graphene dispersed in the epoxy resin, and the mechanical properties of the material are greatly improved. In the present work, graphene oxide with good compatibly was used to increase the toughness. The result shows that graphene oxide can significantly improve the toughness of epoxy resin matrix composites.

\section{Experimental Method}

2.1. Materials. Natural graphite (CP, ignition residue $\leq 0.15 \%$, granularity $\leq 30 \mu \mathrm{m}$ ) was provided by Sinopharm Chemical Reagent Co., Ltd.

Hydrochloric acid (36\%-38\%) and concentrated sulfuric acid (98\%) were obtained from Xi’an San Pu Fine Chemical Plant.

Potassium permanganate $\left(\mathrm{KMnO}_{4}, \mathrm{AR}\right)$ and sodium nitrate $\left(\mathrm{NaNO}_{3}, \mathrm{AR}\right)$ were purchased from Shanghai $\mathrm{Su} \mathrm{Yi}$ Chemical Reagent Co., Ltd.

The epoxy resin E-51 and curing agents were used for this investigation. The epoxy resin E-51 obtained from Wuxi Blue Star resin factory is the diglycidyl ether of bisphenol A resin with an average epoxy value $\mathrm{Ev}=0.51 / 100 \mathrm{~g}$. Methyltetrahydrophthalic anhydride (MeTHPA) as the curing agent was produced by Pu Yang Huicheng Electronic material Co., Ltd. 
2.2. The Preparation of Graphene Oxide. The graphene oxide was obtained by pressurized oxidation [8]. Put a certain amount of $\mathrm{NaNO}_{3}$, natural graphite, and concentrated sulfuric acid (mass ratio is 1:1:50) into the hydrothermal reactor, add $\mathrm{KMnO}_{4}$ slowly under ice water, and tighten the kettle quickly. Freeze for $2 \mathrm{~h}$ under $0^{\circ} \mathrm{C}$ and place it in the oven for $2.5 \mathrm{~h}$ under $110^{\circ} \mathrm{C}$. After it cools, open the kettle, pour it into deionized water, mix it to dilute, and add proper amount of $\mathrm{H}_{2} \mathrm{O}_{2}, \mathrm{HCl}$ to wash. The water suspension is centrifuged centrifuge $5 \mathrm{~min}$ under $8000 \mathrm{rpm}$ and discard the supernatant. Dialyze the underlayer deposition for three days in the dialysis bag and then dry it in the oven under $80^{\circ} \mathrm{C}$. Ultrasonically disperse the dried solid for $30 \mathrm{~min}$ in deionized water at a concentration of $2 \mathrm{mg} / \mathrm{mL}$ and make it become uniformly dispersed liquid which is, namely, graphene oxide aqueous solution. The aqueous solution is dried the dispersed liquid in the oven under $80^{\circ} \mathrm{C}$, grind to obtain the graphene oxide.

2.3. Preparation of GO/Epoxy Resin Composites. The graphene oxide was added to the epoxy resin at $80^{\circ} \mathrm{C}$ in order to lower the viscosity of the epoxy resin. Filler (weight fractions ranging from $0 \mathrm{wt} \%$ to $1 \mathrm{wt} \%$ ) was dispersed in the epoxy resin and was sonicated for $30 \mathrm{~min}$. After cooling to room temperature, the hardener was added to the homogenous mixture and was compounded for another $10 \mathrm{~min}$. The mixture was then taken into a preheated glass mold coated with the mold release agent. The mold enclosed mixture was degassed at $80^{\circ} \mathrm{C}$ under vacuum for $10 \mathrm{~min}$ to remove bubbles. The mixture was cured at $80^{\circ} \mathrm{C} / 1 \mathrm{~h}+120^{\circ} \mathrm{C} / 3 \mathrm{~h}+$ $140^{\circ} \mathrm{C} / 3 \mathrm{~h}$ to complete the crosslink reaction. After being cured completely, the samples were incised according to relevant standard.

2.4. Measurements and Characterizations. The impact tests were performed at room temperature using an impact tester (XCJ-400) according to ASTM-D256. The sample size is $80 \mathrm{~mm} \times 10 \mathrm{~mm} \times 4 \mathrm{~mm}$. The flexural properties were measured using a universal testing machine under the three-point loading scheme (ASTM-D790). The sample size is $80 \mathrm{~mm} \times$ $15 \mathrm{~mm} \times 4 \mathrm{~mm}$. Ten specimens of each composite were tested and the mean values and standard deviations were computed.

Dynamic mechanical analysis (DMA) was performed with a DMAQ800 dynamic analyser at a heating rate of $5^{\circ} \mathrm{C} \mathrm{min}{ }^{-1}$. Samples were heated from $25^{\circ} \mathrm{C}$ to $200^{\circ} \mathrm{C}$ at a fixed frequency of $1 \mathrm{~Hz}$.

Scanning electron microscope (SEM) examinations were observed on the JMS-6480 instrument (Japan's electronics) $\mathrm{SEM}$ at $5.0 \mathrm{kV}$. The specimens were coated with gold vapor to make them conducting.

\section{Results and Discussion}

Impact strength is the ratio of the energy absorbed and the original cross-sectional area of the sample in the impact damage process, which can be used to evaluate impact resistance of material or judge material's brittleness and toughness and thus impact strength also known as impact toughness.

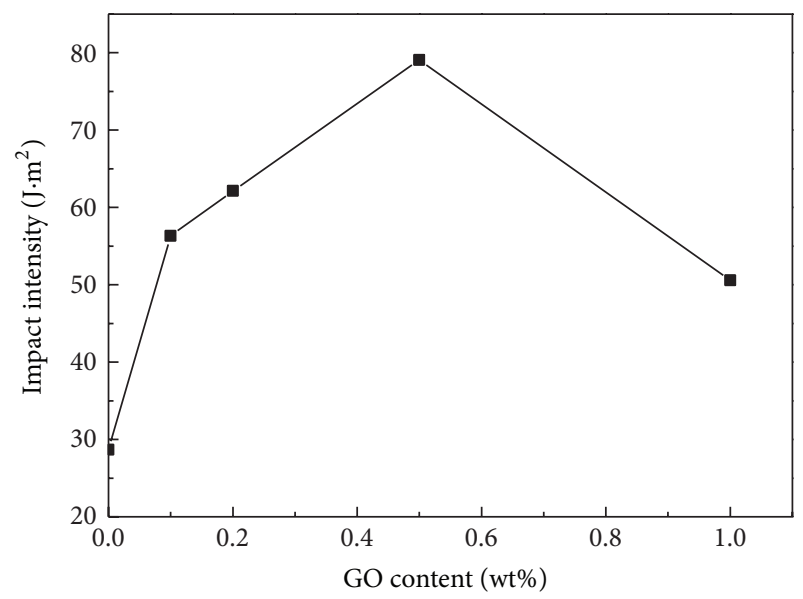

FIGURE 1: The effects of the graphene oxide with different content on the impact strength of the epoxy resin composites.

Figure 1 shows the effects of the graphene oxide with different content on the impact strength of the epoxy resin composites. The figure shows that while the impact strength of pure epoxy resin is $28.66 \mathrm{~kJ} / \mathrm{m}^{2}$, the content of graphene oxide is $0.1 \mathrm{wt} \%, 0.2 \mathrm{wt} \%, 0.5 \mathrm{wt} \%$, and $1 \mathrm{wt} \%$ and the impact strength of epoxy resin composite material is $56.34 \mathrm{~kJ} / \mathrm{m}^{2}, 62.16 \mathrm{~kJ} / \mathrm{m}^{2}$, $79.07 \mathrm{~kJ} / \mathrm{m}^{2}$, and $50.59 \mathrm{~kJ} / \mathrm{m}^{2}$ that increase up to $97 \%, 117 \%$, $176 \%$, and $77 \%$, respectively, which indicates that graphite oxide can significantly improve the toughness of epoxy resin. With the increase of graphite content, the impact strength first increases and then decreases. When the content of graphene oxide is $0.5 \%$, the improvement of the impact strength is up to the best, namely, $176 \%$. Since there are lots of oxygen-containing functional groups, graphene oxide has good compatibility with epoxy resin and well dispersibility in epoxy resin. The large specific surface area of graphene oxide and the high mechanical strength of the pleat on its surface greatly enhance the toughness of epoxy resin composite material. The impact strength increases with lower amplitude when the graphene oxide content is $1 \mathrm{wt} \%$, which probably due to the dispersibility of graphene oxide in epoxy resin decreases with the increase of graphene oxide content, agglomeration, and stress concentration appears; thus, the impact strength decreases.

The flexural modulus, the ratio of bending stresses, and the deformation caused by bending refer to the ability of antibending deformation of material in elastic limit. The flexural strength is the maximum stress a material can bear under the specific deflection or the bending load when material breaks. Figure 2 gives the effects of the graphene oxide with different content on the flexural strength and flexural modulus of the epoxy resin composites. As seen in the figure, the flexural modulus of pure epoxy resin is $3033 \mathrm{MPa}$. When the content of graphene oxide is $0.1 \mathrm{wt} \%, 0.2 \mathrm{wt} \%$, the flexural modulus has little difference with that of pure epoxy. But while the content increases to $0.5 \%$, the flexural modulus is minimum, which further prove that graphene oxide improves the toughness of epoxy resin composites. The bending strength of pure epoxy resin is $114.33 \mathrm{MPa}$. 


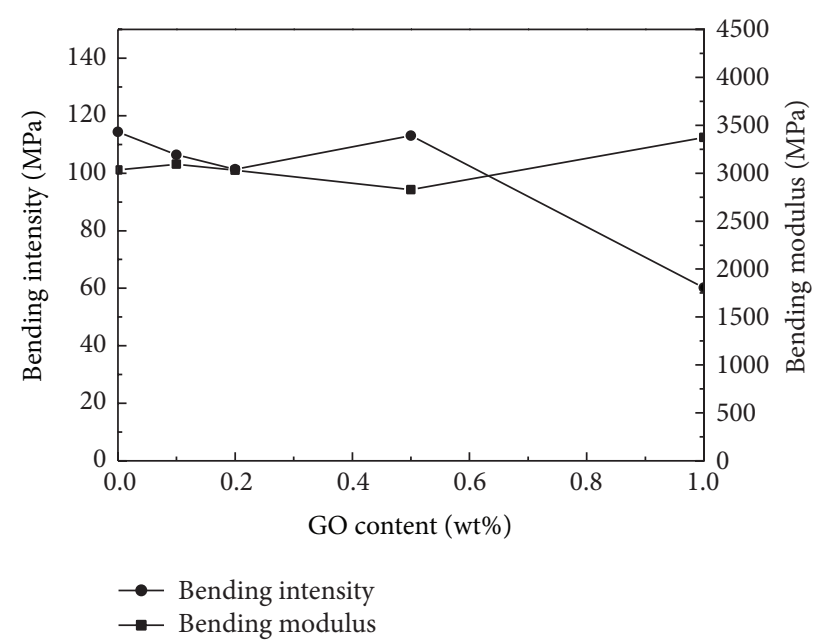

FIgURE 2: The effects of the graphene oxide with different content on the flexural strength and flexural modulus of the epoxy resin composites.

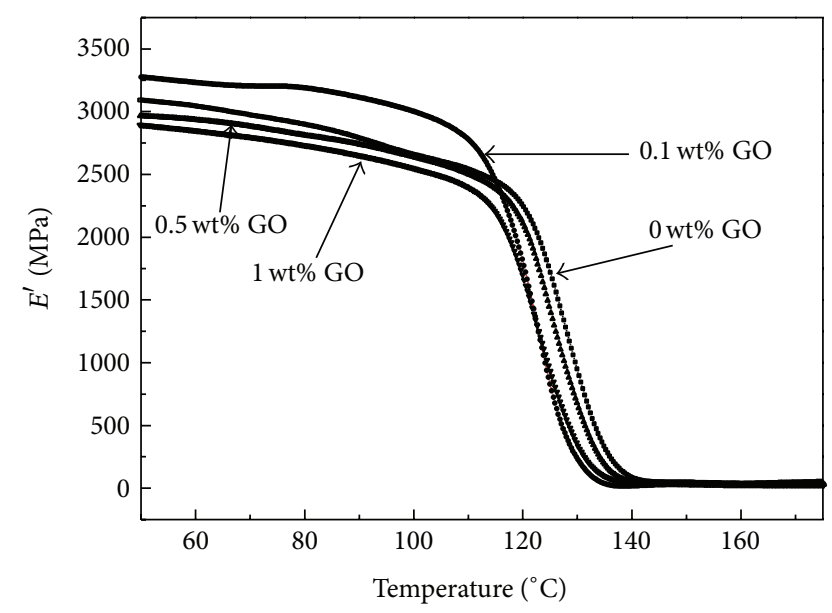

FIGURE 3: The relationship between the temperature and the storage modulus of graphene oxide/epoxy resin composite material.

When graphene oxide content is $0.1 \mathrm{wt} \%, 0.2 \mathrm{wt} \%, 0.5 \mathrm{wt} \%$, and $1 \mathrm{wt} \%$, the bending strength of epoxy resin composites is $106.382 \mathrm{MPa}, 101.351 \mathrm{MPa}, 113.07 \mathrm{MPa}$, and $60.25 \mathrm{MPa}$ and decreases to $7.0 \%, 11.4 \%, 1.0 \%$, and $47.3 \%$, respectively. This indicates that graphene oxide does not make the strength of epoxy resin decrease significantly while increasing its toughness. The toughness is best when the content of graphene oxide is $0.1 \mathrm{wt} \%$ but bending strength decreases only $1.0 \%$. The toughness and rigidity of epoxy resin based composite are worse than pure epoxy resin matrix when the content of graphene oxide is $1 \mathrm{wt} \%$ due to uneven dispersion.

The dynamic mechanical properties are conducted in order to study the effect of graphene oxide content on the rigidity and damping properties of epoxy resin based composite. Figure 3 gives the relationship between the temperature and the storage modulus of graphene oxide/epoxy resin composites. It can be seen from Figure 3 that low temperature

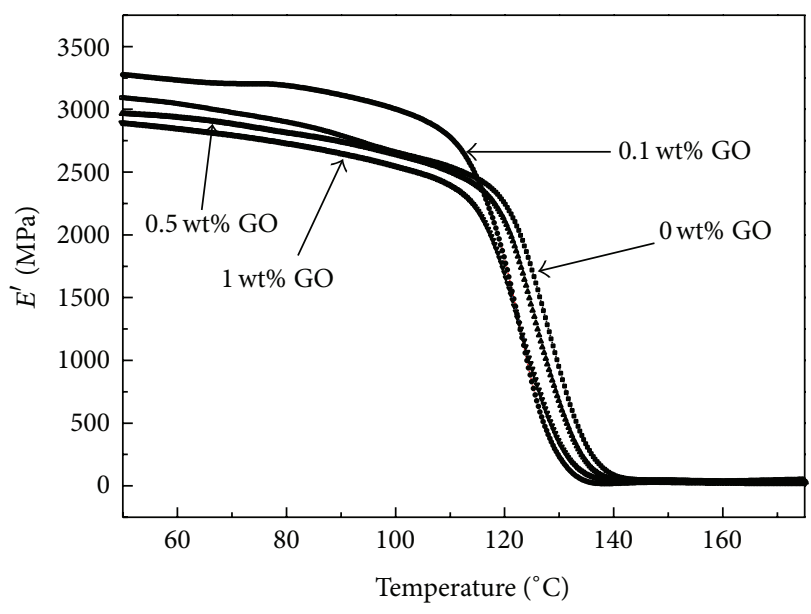

Figure 4: The relationship between the temperature and the loss modulus of graphene oxide/epoxy resin composites.

zone corresponds to the glassy state of the polymer, so the storage modulus is very high at this region and decreases slowly with the increase of the temperature when the polymer chain segments are frozen. Deformation is mainly caused by the bending of the chemical bond in the polymer chain, and the corresponding mechanical strengths reach the best at the low temperature. Free segments begin to move with the increases of the temperature and the excess energy dissipates into heat. In a certain temperature range, this change reaches the maximum, the storage modulus decreases rapidly, and this temperature range is the glass transition region. In the transition zone, mechanical strength significantly and rapidly decreases. When the temperature is higher than the transition point, the material mainly shows the viscoelasticity because molecular chain can move freely; therefore, the storage modulus representing rigidity and elasticity is not high. Before reaching the glass transition temperature, there is slight decrease of storage modulus entirely with increase of the content of graphene oxide, which confirms the decrease of the bending strength mentioned above. While the flexible graphene oxide improves the toughness of the composite, the rigidity decreases slightly at the same time due to the network relaxation. Besides, the glass transition temperature of the composites decreases because of the bad thermal stability of graphene oxide containing oxygen functional groups.

The loss modulus, on behalf of the viscidity of material in the viscoelasticity, reflects the energy consumption which convert to heat energy during the deformation of material [9]. From Figure 4, it can be known that before the glass transition temperature, pure epoxy resin matrix mainly shows the elasticity of the material; thus, the loss modulus is small and changes a little. With temperature increasing, free segments begin to move and the excess energy dissipates into heat. The transition reaches biggest in the glass transition temperature zone and the loss factor curve appears the peak. When the temperature is higher than $\mathrm{Tg}$, the polymer is in the high elastic state, segments can move and slide freely, and the internal friction and the loss modulus decrease. After adding the graphene oxide, the loss modulus of the composites is 


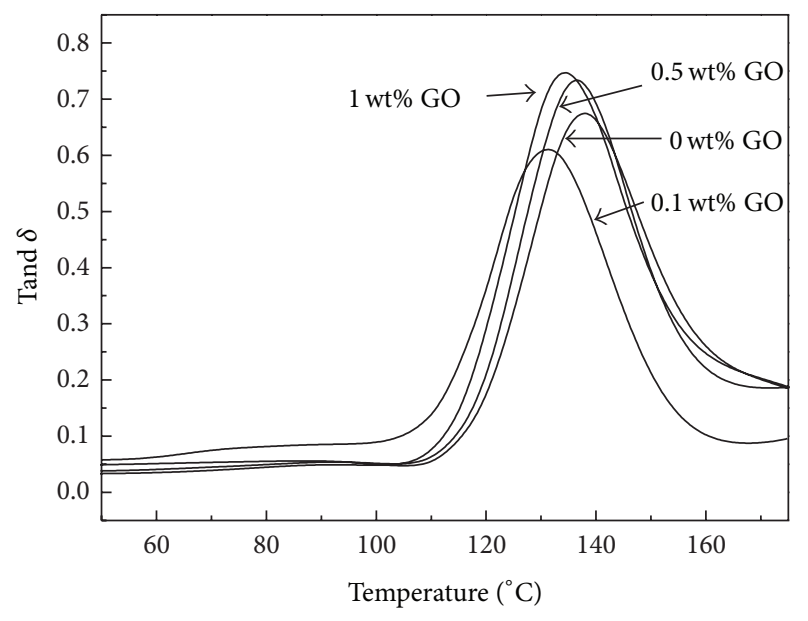

FIgURE 5: The relationship between the temperature and the loss factor of graphene oxide/epoxy resin composites.

small relatively before reaching the glass transition temperature and increases slightly with increasing temperature. The loss modulus rises sharply and reaches the peak at the glass transition temperature. In addition, since the addition of graphene oxide makes the molecular chain network structure relax, the glass transition temperature moves to the lower temperature region.

Figure 5 shows the relationship between the temperature of graphene oxide/epoxy resin composite material and the loss factor. The loss factor Tand $\delta$ is the ratio value of the loss modulus $E^{\prime \prime}$ and the storage modulus $E^{\prime}$. Figure 5 shows that the glass transition temperature of pure epoxy resin corresponding to the loss factor peak value is $140^{\circ} \mathrm{C}$ and loss factor is 0.65 . The glass transition temperature moves to the lower temperature zone and the loss factor increases with the addition of graphene oxide. When the content of graphene oxide is $0.5 \%$, the loss factor Tand $\delta$ reaches the biggest, namely, 0.72 , and the glass transition temperature is the lowest. This indicates that while increasing the toughness of epoxy resin, graphene oxide could transform the applied mechanical energy into other forms of energy promptly and thus improve the damping property of epoxy resin. When the content is $1 \mathrm{wt} \%$, the damping performance get bad and the mechanical properties decrease, which probably causes by the poor dispersion of graphene oxide.

Figure 6 is the impact fracture morphology of the GO/ epoxy resin composites. Figure 6(a) shows the typical brittle fracture of pure epoxy resin whose section is neat and the crack appears smooth river pattern zones, while in Figure 6(b) the impact sections of GO/epoxy resin composites are irregular in size and there are more failure surfaces. The epoxy groups of graphene oxide make splitphase graphene oxide and the matrix have excellent interface compatibility. When the system loaded, homogeneously dispersed graphene oxide in the composites could initiate and terminate cracks under the stress. The system consumes lots of energy. In the morphology of fracture, impact and cleavage fracture surfaces are rough, the failure surfaces are more, energy consumption is larger, and the appearance of

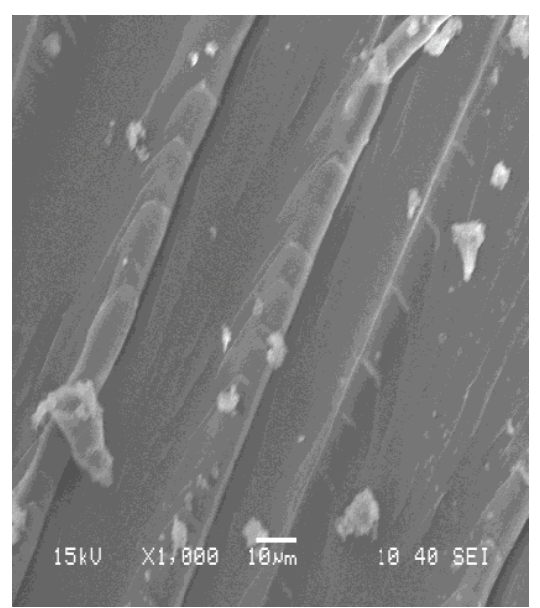

(a)

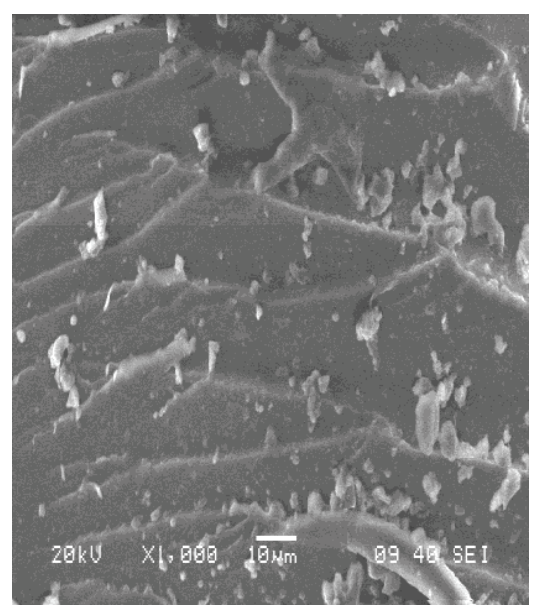

(b)

FIgURE 6: The SEM images of the surface appearance of the abrasion of (a) pure epoxy resin composites and (b) GO/epoxy resin composites.

ductile failure indicates the achievement of the purpose of toughening.

\section{Conclusion}

The graphene oxide was obtained by pressurized oxidation method and the polymerization. GO/epoxy resin composites were successfully prepared by casting. Graphene oxide significantly enhances the toughness of the composites while it does not decrease the strength of the composites obviously. As the graphene oxide content is $0.5 \mathrm{wt} \%$, the impact intensity of the composites reaches the largest value by $176 \%$ compared with pure epoxy resin matrix, and the damping capacity is obviously improved.

\section{Conflict of Interests}

The authors declare that there is no conflict of interests regarding the publication of this paper. 


\section{Acknowledgments}

The authors greatly acknowledge the Special Fund for Basic Scientific Research of Central College, Jiangsu Provincial Cooperative Innovation Fund of China (Grant no. BY2012178), and National Natural Science Foundation (51402132).

\section{References}

[1] K. S. Novoselov, A. K. Geim, S. V. Morozov et al., "Electric field in atomically thin carbon films," Science, vol. 306, no. 5696, pp. 666-669, 2004.

[2] M. Hirata, T. Gotou, S. Horiuchi, M. Fujiwara, and M. Ohba, "Thin-film particles of graphite oxide 1: high-yield synthesis and flexibility of the particles," Carbon, vol. 42, no. 14, pp. 2929-2937, 2004.

[3] Q.-H. Yang, W. Lu, Y.-G. Yang, and M.-Z. Wang, "Free twodimensional carbon crystal-single-layer graphene," Xinxing Tan Cailiao/ New Carbon Materials, vol. 23, no. 2, pp. 97-103, 2008.

[4] S. Stankovich, D. A. Dikin, R. D. Piner et al., "Synthesis of graphene-based nanosheets via chemical reduction of exfoliated graphite oxide," Carbon, vol. 45, no. 7, pp. 1558-1565, 2007.

[5] R. van Noorden, "Moving towards a graphene world," Nature, vol. 442, no. 7100, pp. 228-229, 2006.

[6] J. Liang, Y. Wang, Y. Huang et al., "Electromagnetic interference shielding of graphene/epoxy composites," Carbon, vol. 47, no. 3 , pp. 922-925, 2009.

[7] D. R. Bortz, E. G. Heras, and I. Martin-Gullon, "Impressive fatigue life and fracture toughness improvements in graphene oxide/epoxy composites," Macromolecules, vol. 45, no. 1, pp. 238-245, 2012.

[8] C. Bao, L. Song, W. Xing et al., "Preparation of graphene by pressurized oxidation and multiplex reduction and its polymer nanocomposites by masterbatch-based melt blending," Journal of Materials Chemistry, vol. 22, no. 13, pp. 6088-6096, 2012.

[9] Y.-P. Zheng, J.-X. Zhang, Y.-H. Xu, F. Dai, and B. Wang, "Moisture absorption mechanism of epoxy resin matrices through positron annihilation technique," Polymeric Materials Science and Engineering, vol. 25, no. 8, pp. 110-113, 2009. 

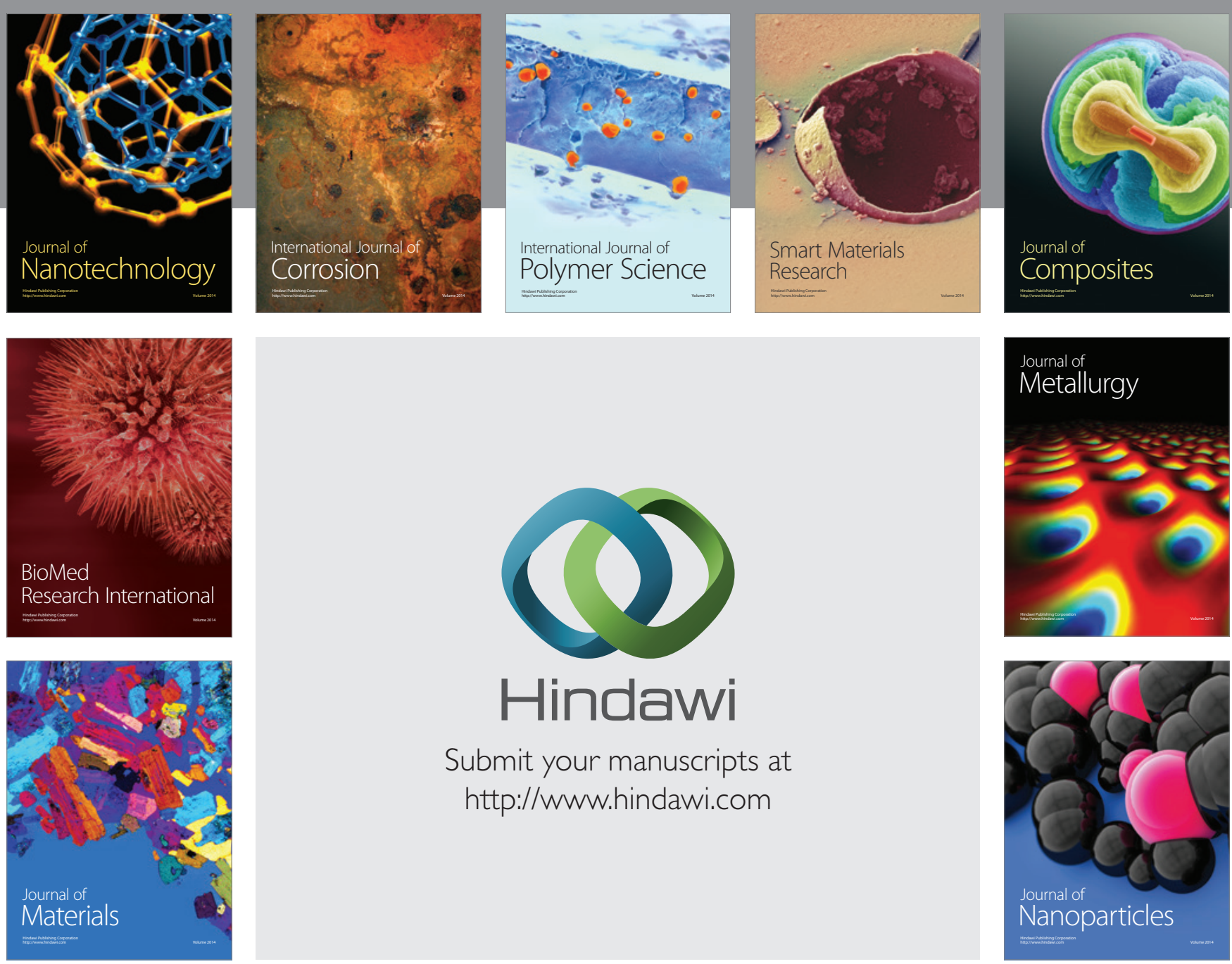

Submit your manuscripts at http://www.hindawi.com
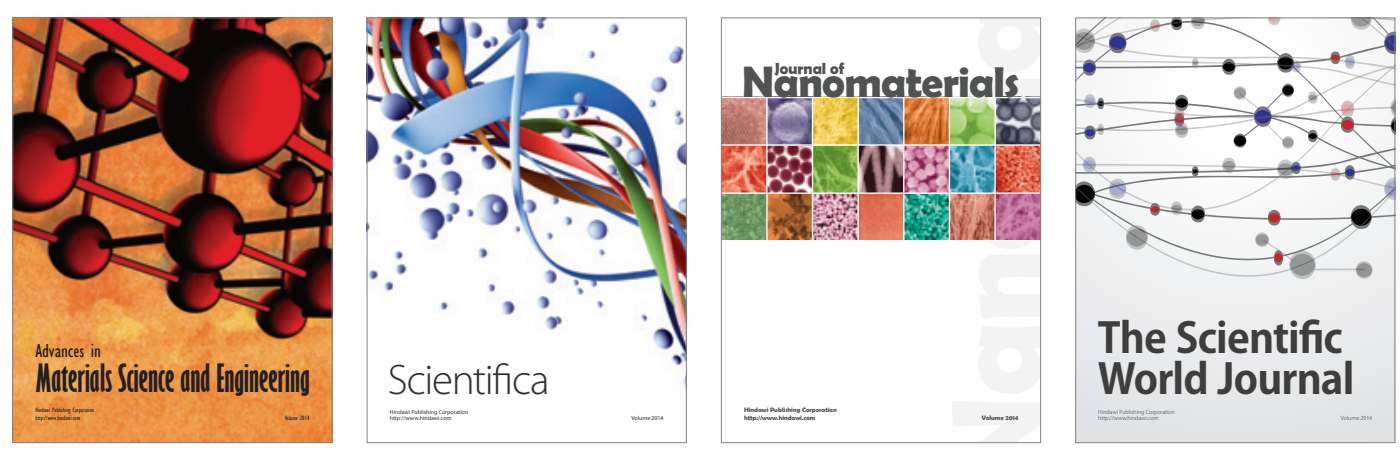

\section{The Scientific World Journal}
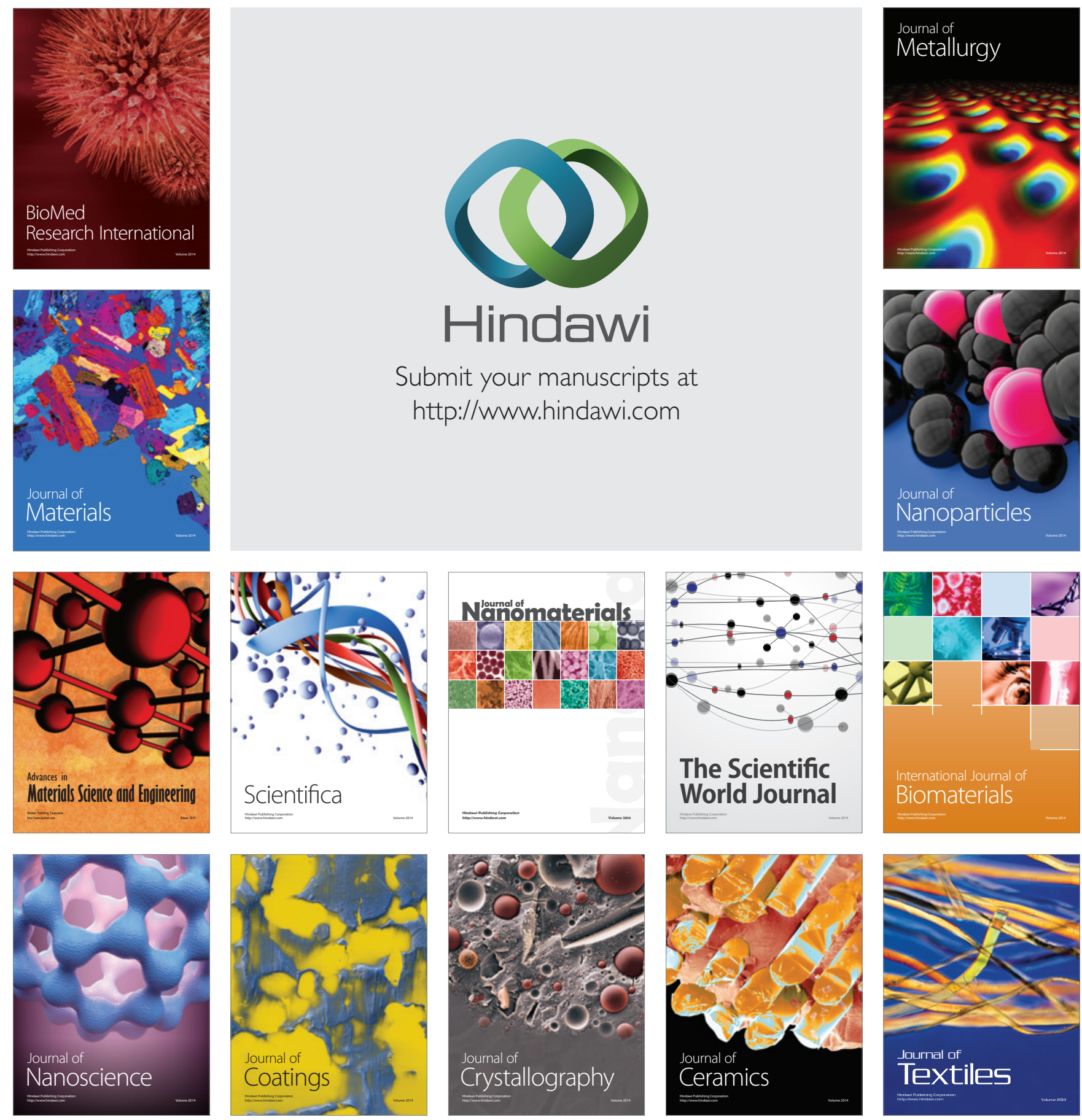